\title{
Online Book Marketing System
}

\author{
Ping Wang
}

\section{College of Information Engineering, Jiangxi University of Technology, Jiangxi Nanchang}

\author{
Keywords: Convenience; Browse by category; Shopping cart; ASP.NET
}

\begin{abstract}
In order to achieve the convenience of online book marketing system, the online book marketing system was developed. The system classified the books, and it's convenient for the users to browse; users can get the books they need any time and any place without being affected by the time and place. The main functions of the online book shopping system are that user first registers member of the website, and subscribes to the books they need by the shopping cart. User can also directly query the books in the address search bar by inputting relevant information. The system has good stability and security by adopting ASP.NET technology of Microsoft, database SQL Server2000 and the B/S structure.
\end{abstract}

\section{Overview}

The significance of developing online book marketing is to combine the book marketing and network, and promote the book marketing into a new stage; the online book marketing has great advantage compared with the conventional publications. In traditional publication industry, the movement of publication content or information is always accompanied by the movement of material carrier in the whole or part of the process of creation, publication, reception and usage. Under the internet environment, the author, editors and publishers, sellers and readers are all on the internet; there is only information flow but no logistics.

In the whole process of business activity, online book marketing greatly improves the efficiency of business activity through combination of people and electronic communication, and reduces the unnecessary links. The traditional manufacturing industry has entered the era of small batch and multi-variety, and "zero-inventory" becomes possible; traditional retail industry and wholesale business created the new model of "non-shop" and "online marketing"; all kinds of online services have provided brand new service mode for traditional service industry. Therefore, there is much space to develop the online-book, and it's an effective way to promote economic development.

As part of the computer application, the combination of traditional book marketing and network through internet can allow people to search information and subscribe to books, which are a kind of revolutionary innovation on traditional book marketing in a sense, especially by making use of the characteristics of e-commerce, such as security, high-efficiency and convenience. For example, it is convenient for search, high in reliability, less error-prone, low in cost, etc. These advantages can greatly increase efficiency, and are significant conditions for scientific and normalized management and integration with the world. The paper achieved the online book marketing system based on the design.

\section{System analysis}

Preliminary survey on system. The main objective of preliminary survey on system is to analyze the necessity and possibility of new project development from the perspective of system analysts. First, analyze the existing system, which is the major source of information. Determine the design objective and model of new system through analyzing the advantages and disadvantages of existing system, which made the design of system easier and can be modified at any time. 
The study on existing system:

China book network has the following simple functions:

Login: manage the user through the login module;

Registration: users can log in after registration, and save personal information;

Query: mainly search books through fuzzy search;

Shopping: add the books into the shopping cart;

Settlement: pay the bill

Feasibility study. The main task in feasibility study phase is to judge whether the new system can be achieved or worth realization on the basis of preliminary survey, and avoid the situation that the system cannot be achieved or the new system has no practical meaning after spending lots of manpower and material resources; the feasibility study on the new system is to determine whether the system is feasible with lowest price and in as short a time as possible.

Run feasibility study

With the popularization and promotion of computer, more and more people have mastered the basic usage and skills of computer, and network has become the indispensable means of communication for most of computer users. Especially in recent years, the development of computer network has gone beyond people's imagination, so the development of online marketing system is possible. The cyber citizens show great interest and enthusiasm to the development of new system, put forward various good suggestions and requirements, enrich the creation inspiration, solve the problems of online book marketing, which are all the external reasons for the development of system.

According to the above analysis, the development of new online book marketing system is totally necessary and feasible.

Demand analysis. The demand analysis includes user demand and function demand two aspects.

User demand:

At first, the online book marketing system is open to all the cyber citizens, so the website should meet the demand of all the cyber citizens. The website should be simple and friendly in the page setting, easy for operation; most importantly, the security of subscriber data should be guaranteed, and the invasion of personal privacy should be avoided; the users would like to register and buy the books they need if the security is guaranteed, so the security of online book marketing system is very important. The security is the key factor for the successful system; otherwise it would be a useless system.

Function demand

According to the demand analysis of users, the main functions of system should include login, registration, query, shopping and settlement; these functions might meet the basic demand of the system.

\section{Database design}

The design of database entity. The design of database entity needs to consider the characteristic of centralized data services, consider the problems such as data dependence, data redundancy and data response according to the database design model, and take into account the difficulty of function realization.

The following is the entity design:

1. The Customers table stores the log-on message of members, which includes user name, password, email, address, phone number, etc. In the Customer table, due to the same user name, the CustomerID is designed as master key, which is the auto-increment type. In order to facilitate the memory, users use the CustomerEmail as the unique identifier when they $\log$ in. The users' identity can be determined by CustomerEmail and CustomerPassword. The 
Customers table stores the log-on message of members such as user name, password, email, address, phone number, etc. In the Customer table, due to the same user name, the CustomerID is designed as master key, which is the auto-increment type. In order to facilitate the memory, users use the CustomerEmail as the unique identifier when they log in. The users' identity can be determined by CustomerEmail and CustomerPassword.

2. The book table stores the book information which includes the book number, name, type, ISBN, URL address, price and content introduction, in which BookID is the master key of the table, and CategoryID is the foreign key of Categories. The table of CategoryID can facilitate the query and management and be shown according to the book type in the page.

3. The classification chart classifies the books, and together forms the father-son table with Books table.

4. Shopping cart table records the shopping information of users every time. In practice, users may not buy only one book every time; CardID records when users add books into the shopping cart each time, so one shopping cart may have many records. In practical design, RecordID is the auto-increment master key rather than the CardID, so CartID distinguishes each shopping and RecordID distinguishes the book of the shopping. It is very important to understand the business flow of the system.

It is permissible for non-registered users to buy books; the system automatically generates one random character string for record, so the books in the shopping cart can be transferred to the official account when the users settle accounts.

In addition, Quantity records the quantity of each shopping; in the design of database, the default value is 1 , and it reduces the input of user.

5. The order sheet will be generated when the users settle accounts. It records the purchase situation of users each time. The default-generated date is the very day; Shopdate value is within 1 to 3 days by using random function.

6. The order detail table is similar to the "history record", which is convenient for the users to check their own previous purchase situation. OrderID and BookID are its master key, and they codetermine one piece of history record.

7. Comment table records the evaluation of books. It needs to record the CustomerEmail and BookID of users, shows the evaluation of books from users, records the comment registration by Rating, and content by Comments.

The design of data access layer. The data access layer is the middle tier that links the top and bottom layer of database and its leading role is reliability; the database access layer won't change, and the user level won't change even if the database changes.

\section{Overall design}

Object design. 1. Strong practicality: in accord with the habit of practical operation flow; easy learning and use, and friendly user interface; suitable for the usage requirements of all-level users;

2. Advanced program structure: the application of contemporary advanced software programming can increase lifespan and be easy for maintenance and management;

3. The application of modular design: the system has good expandability in order to adapt to the development of different stage;

4. Simple operation and convenient maintenance: every system has the function of system maintenance, and can automatically maintain the changeable projects;

5. Strong query function: the book can be queried by name

6. Realization of .net application: three-layer structure can be realized by network programming language (such as ASP, JSP, etc.); the better way is to realize by c\# or particular class of java. The paper was to construct three-layer application by adopting c\#. The function 
of database interaction and html web script generation are realized through c\#, which saves time for the ASP compiling from WebSerrve, and greatly improves the application speed of large-scale application. The paper made a useful attempt.

The design of system function module. Follow the principle of module independence; the descriptions of each function module are as follows:

1. User login: it is for the login of users and the management of accounts; users can buy books only after login;

2. Registration module: register users and save the specific information of users;

3. Book query: query relevant books through fuzzy search;

4. Shopping: add books into the shopping cart;

5. Settlement: settle accounts.

\section{Detailed design}

Module introduction. Design of login module.

The online book marketing system allows anybody to browse, but only registered members can buy the books of the website.

\section{Program debugging and testing}

Debugging. The purpose of system debugging is to find the errors in the program and system and correct them. The debugging of system should include program debugging, function debugging (partial adjustment), and overall adjustment that includes grammar debugging and logic check. Besides the normal data, testing data should also use some abnormal data in order to test the correctness of the program.

1. Debug with normal data. For example, give the users some hints when entering information.

2. Debug with wrong data. For example, input the wrong length or type of the user name deliberately at registration, and the system will prompt the error.

Partial debugging.

Debug all the programs in one function in order, which is to guarantee the correctness of internal control relationship and data content, and test the running efficiency of the module. For example, test whether individual module can be realized.

Overall debugging

Combine the master control scheduler and function module and debug; make feasibility test to the various possible usage patterns and the circulation of its combination in the software, such as user registration, user login, query, shopping and settlement.

Test. In the online book marketing system, the testing methods include manual testing and mechanical testing. The manual testing is done by individual review, namely after writing the source program, it is tested by the programmer; however, the testing method has its shortcoming, for example, due to preference to his own program, the habitual mistakes are difficult to be found by programmers; what's more, it is not easy to correct the mistakes if the function is misunderstood.

Run the results. The program can be run, has complete functions, and can realize the functions such as login, registration, query, shopping, settlement, etc.

\section{Acknowledgements}

This work was financially supported by project of Technology Department of Jiangxi Province [No 20143BBM26048] and the project of Technology Department of Jiangxi Province [No 2013BBE50051] also gives us lots of help. 


\section{References}

[1] Sun Yong and others. Modern software engineering. First edition. Beijing: Beijing Hope Electronic Press, 2002: 2 3

[2] Sun Sancai, Xu Xunyin. Master C\# and ASP. Second edition. NET. Beijing: China Railway Press, 2003:3 4

[3] Tang Li. Prospect of online bookstore, Beijing Daily, fifth edition in the year of 2006(56 editions in all)

[4]Bruce Billy Brown Karl The 3th New York SHOPPINEG ON LINE

[5] Li Gang. Wang Haoliang. Course of Visual C\# program design. Fourth edition. Beijing: Tsinghua University Press, 2003:23 24

[6] Zhang Xiaopan, Tian Ye. 50 Classic examples of ASP.NET. Second edition. Beijing: Science Press, 2004:15 17

[7] Wang Rong. Stored procedure of SQLServer2000 and XML programming. Third edition. Beijing: Electronic Industry Press, 2004.26 28 